\title{
Now the future, we see our dreams: artificial intelligence in drug discovery
}

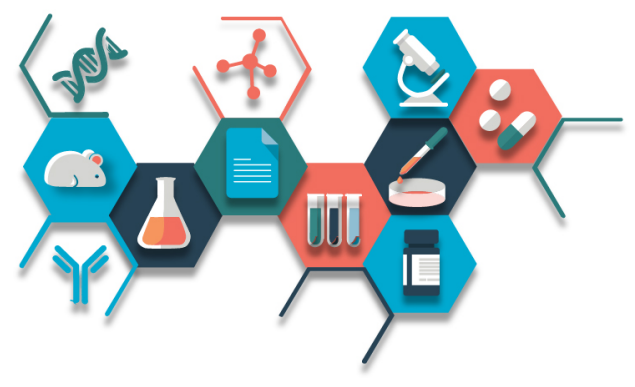

Rae Lawrence*,1

${ }^{1}$ Drug Discovery Unit, Cancer Research UK Manchester Institute, The University of Manchester, Alderley Park, SK10 4TG, UK

*Author for correspondence: Tel.: +44 161306 6280; rae.lawrence@cruk.manchester.ac.uk

\section{"the success of any computational approach hinges on the quality of the data used to teach or train the systems"}

First draft submitted: 21 August 2019; Accepted for publication: 10 October 2019; Published online: 21 October 2019

Keywords: artificial intelligence $\bullet$ computational chemistry $\bullet$ drug design $\bullet$ drug discovery $\bullet$ machine learning

The rapid progression toward routinely using artificial intelligence (AI) in drug discovery continues to be impressive; our group, like most modeling teams, is keenly interested in exploring the potential of AI to support our efforts. Recognizing that the computational chemistry community has occasionally overpromised and underdelivered, we must temper expectations for what is achievable today with the available data versus what may be achievable in the future. While there is healthy skepticism about whether a machine can magically churn out a successful clinical candidate, there is an abiding faith that AI methods will help reduce the time and costs associated with bringing a drug to the clinic and eventually to the patients that need it.

\section{"I'm sorry Dave, I'm afraid I can't do that."- HAL9000. 2001: A Space Odyssey [1].}

For many of us of a certain vintage, few moments of cinematic genius invoke such mental discomfort as those nine softly spoken words by HAL-9000 in his soothing, comforting voice with his unrelenting, unblinking, red dot eye. The notion that a machine can be programmed to consciously learn and develop independent intelligence and decision-making skills was terrifying. Fast-forwarding some 50 years since the release of 2001, A Space Odyssey, we are at the cusp of routinely incorporating AI into medicinal chemistry design and are already successfully applying machine learning (ML) in the design and optimization of new drugs. It is exciting, not scary.

Let's rewind, for a moment, to the simple, grungy days of the late 1990s; with the release of faster processors and impressive improvements in computational chemistry algorithms, some computational chemistry experts became arrogant about the value that could be delivered to medicinal chemistry programs. "Yes - of course we can deliver an in silico designed drug in open intellectual property space that will be easy to make, potent, selective, efficacious, good ADME/T (absorption, distribution, metabolism, excretion, toxicity), have no off-target liabilities and all set for the clinic. Easy peasy lemon squeezy." Everyone believed us, and why would they not? Computational chemistry was the sun that was going to illuminate drug discovery chemistry.

\section{We were young and naive. \\ In our youthful folly, we were overconfident. \\ And mistaken. \\ But in our dreams, we saw the future. \\ Now the future, we see our dreams. \\ - R Lawrence}

Having learned our lesson, many of us are hesitant to declare AI as the savior of our field. That being said, as hardware and algorithms (and data quality) improve, it is abundantly clear that AI is something that is rapidly becoming a critical part of our workflows, and over time, will evolve until it is as fundamental to discovery chemistry as chromatography and structure-activity relationships. 
The cost to develop a drug, of which most fail in the clinic, exceeds $\$ 2$ Billion USD and a decade's worth of time. In recent years, virtually all major pharmaceutical companies took the plunge and invested enormous amounts of time and money into AI to try to work smarter and to decrease timelines and costs associated with getting a drug from 'candidate to mandate'. Some have invested in internal resources; others, by collaboration with external AI-focused companies [2]. According to a recent report, the AI in healthcare market was worth $\$ 667$ Million in 2016, and anticipated to reach $\$ 8$ billion by 2022 [3].

There are an ever-increasing number of dedicated AI companies working in the drug discovery arena, including BenevolentAI, GTN (both London, UK), and Exscientia (Dundee, UK). Each have had initial technical successes and have secured investor capital and pharmaceutical industry collaboration to continue their efforts to perfect their approaches to accelerate the drug discovery and development cycles, reduce spending and create effective drugs [4-7].

At the Cancer Research UK (CRUK) Manchester Institute, we also have a blossoming interest in using AI solutions to help us discover new drugs in our Drug Discovery Unit. Our group is funded by CRUK, which is the UK's largest charity [8]. CRUK's vision is to 'bring forward the day that all cancers are cured' by fostering and funding research that will, within 20 years, result in $75 \%$ of patients surviving. While CRUK researchers work to find diagnostics, preventions, treatments and cures for all types of cancer, there is a special interest in hard-to-treat cancers like lung, pancreatic, esophageal and brain cancers where there is unmet patient need. These cancers often involve extremely challenging targets often requiring new strategies and embracing new technologies to drive research forward.

CRUK-MI's Drug Discovery Unit (DDU) was purpose built within the CRUK Manchester Institute to have the ability to take a program from hit identification to preclinical development on challenging targets. Our teams in chemistry, biology, biochemistry and informatics/modeling understand the cyclical relationship and iterative nature of modeling and discovery, and these approaches are applied across all projects. Over the past year, our informatics and modeling team has been boosting capacity and has begun to explore AI options to support our various programs.

In the DDU, our understanding of $\mathrm{AI}$ is that it is a machine or computer that has been programmed to do things that humans can presently do better but tend to be slow at doing. Systems incorporating AI process and integrate massive amounts of data and knowledge and analyze previous decisions; the algorithm applies the learned knowledge to solve new problems using complex problem-solving skills. Ultimately, the AI system will be able to surpass humans in both accuracy and speed through automation of pattern interpretation.

On the other hand, ML (a form of $\mathrm{AI}$ ) extracts knowledge from data to help humans make decisions. ML is something we do routinely in our discovery programs: Quantitative Structure-Activity Relationships (QSAR) are built using ML methods to relate structural and/or physicochemical features of molecules with an observable (e.g., potency), and large collections of compounds are clustered based on their structural similarity. Both clustering and QSAR modelling rely on common ML algorithms!

Ultimately, the success of any computational approach hinges on the quality of the data used to teach or train the systems - as the old saying goes: garbage in, garbage out. Data, such as that regarding structure and assay information, that are housed by publicly available sources like ChEMBL [9] and PubChem [10] are often found to have transposition and curation errors [11]. While strides have been and continue to be made to improve autocuration and error-management for large volumes of data [12-15], errors do creep in, and in modeling, we must be aware of that possibility and ensure that measures are in place to minimize their impact or at least be able to identify data which are spurious.

Our team boasts one of the most technophilic groups of scientists I have had the privilege of working with in the past 20 years; yet, there is healthy skepticism about AI approaches for discovery in our ranks - as there should be [16]. This skepticism is fueled for a number of reasons, including "being burned by compchem before", a lack of core understanding of the technology and preconceived notions about what $\mathrm{AI}$ is and what it can do ("can it make coffee?"). At the same time, there is an emerging feeling of cautious optimism that this just might work; maybe not perfectly, but enough to build confidence.

Fully recognizing that our unit does not have human or hardware resources required to implement DDUowned AI solution, we have sought collaborators working in this space. In the autumn of 2018, we commenced collaboration with a company that specializes in AI solutions (based on deeper learning using quantum mechanical descriptors) for the pharmaceutical industry. Through the relationship with our collaborative partner, it is believed that ultimately our models will be able to generalize across targets to predict activity where there is little or no data 
available. Further, we believe the accuracy is bound to surpass existing models based on chemical fingerprints and traditional ML approaches that are regularly used. Time will tell whether this approach will work.

It is a risky venture, but ultimately, it is one worth taking. AI approaches may very well not live up to our lofty expectations of churning out a clinic-ready drug, but then again, it could give us the key insights we need to reach our ambitious goal of curing all types of cancer in this generation.

Now the future, we are seeing our dreams.

\section{Disclaimer}

The opinions expressed in this work are entirely those of the author and do not necessarily reflect those of the University of Manchester, CRUK Manchester Institute or Cancer Research UK.

\section{Financial \& competing interests disclosure}

R Lawrence is funded by Cancer Research UK (grant number C5759/A17098). The authors have no other relevant affiliations or financial involvement with any organization or entity with a financial interest in or financial conflict with the subject matter or materials discussed in the manuscript apart from those disclosed.

No writing assistance was utilized in the production of this manuscript.

\section{Open access}

This work is licensed under the Creative Commons Attribution4.0 License. To view a copy of this license, visit http://creativecommons.org/licenses/by/4.0/

\section{References}

1. Kubrick S, Clarke AC. 2001: A Space Odyssey. Metro-Goldwyn-Mayer Corp, CA, USA (1968).

2. Fleming N. How artificial intelligence is changing drug discovery. Nature 557(7707), S55-S57 (2018).

3. Cision PR Newswire. Global artificial intelligence in healthcare market 2017-2022: market is expected to reach USD 7,988.8 million at a CAGR of 52.68\% - research and markets (2017). http://www.prnewswire.com/news-releases/global-artificial-intelligence-in-healthca re-market-2017-2022-market-is-expected-to-reach-usd-79888-million-at-a-cagr-of-5268---research-and-markets-300464679.html

4. GOV.UK. Artificial intelligence sector deal (2019).

http://www.gov.uk/government/publications/artificial-intelligence-sector-deal/ai-sector-deal

5. BenevolentAI. BenevolentAI raises $\$ 115$ million to extend its leading global position in the field of AI enabled drug development (2018). https://benevolent.ai/news/announcements/benevolentai-raises-115m-for-ai-enabled-drug-development

6. Exscientia. Celgene and Exscientia enter 3-year AI drug discovery collaboration focused on accelerating drug discovery in oncology and autoimmunity (2019). http://www.exscientia.co.uk/news/2019/3/20/celgene-and-exscientia-enter-3-year-ai-drug-discovery-collaborat ion-focused-on-accelerating-drug-discovery-in-oncology-and-autoimmunity

7. Exscientia. Exscientia achieves molecule discovery milestone as part of GSK collaboration (2019). http://www.exscientia.co.uk/news/2019/4/3/exscientia-achieves-discovery-milestone-in-gsk-collaboration

8. Cancer Research UK (2019). https://www.cancerresearchuk.org/

9. Gaulton A, Hersey A, Nowotka M et al. The ChEMBL database in 2017. Nucleic Acids Res. 45(D1), D945-D954 (2017).

10. Kim S, Chen J, Cheng T et al. PubChem 2019 update: improved access to chemical data. Nucleic Acids Res. 47(D1), D1102-1109 (2019).

11. Tiikkainen P, Bellis L, Light Y, Franke L. Estimating error rates in bioactivity databases. J. Chem. Inf. Model. 53(10), 2499-2505 (2013).

12. Cortes-Ciriano I, Bender A, Malliavin TE. Comparing the influence of simulated experimental errors on 12 machine learning algorithms in bioactivity modeling using 12 diverse data sets. J. Chem. Inf. Model. 55(7), 1413-1425 (2015).

13. Hersey A, Chambers J, Bellis L, Bento AP, Gaulton A, Overington JP. Chemical databases: curation or integration by user-defined equivalence? Drug Discov. Today Technol. 14, 17-24 (2015).

14. Papadatos G, Gaulton A, Hersey A, Overington JP. Activity, assay and target data curation and quality in the ChEMBL database. J. Comput. Aided Mol. Des. 29(9), 885-896 (2015).

15. Mansouri K, Grulke CM, Richard AM, Judson RS, Williams AJ. An automated curation procedure for addressing chemical errors and inconsistencies in public datasets used in QSAR modelling. SAR QSAR Environ. Res. 27(11), 911-937 (2016).

16. Jordan AM. Artificial intelligence in drug discovery - the storm before the calm? ACS Med. Chem. Lett. 9(12), 1150-1152 (2018). 